\title{
Conducting randomised controlled trials with older people with dementia in long-term care: challenges and lessons learnt
}

\begin{abstract}
The characteristics of older people with dementia and the long-term care environment can make conducting research a challenge and, as such, this population and setting are often understudied, particularly in terms of clinical or randomised controlled trials. This paper provides a critical discussion of some of the difficulties faced whilst implementing a randomised controlled trial exploring the effect of a live music program on the behaviour of older people with dementia in long-term care. A discussion of how these challenges were addressed is presented to aid investigators planning the design of similar research and help encourage a proactive approach in dealing with research-related challenges right from project conception. The article is structured according to the three principles of a randomised controlled trial in order to keep experimental rigour at the forefront of this research area.
\end{abstract}

Key words: cross-over design; dementia; methodology; randomised controlled trial; research design. 


\section{INTRODUCTION}

Dementia is a clinical syndrome with a number of different causes. It is characterised by the decline of a person's cognitive functioning, affecting skills in language, memory and perception $^{1}$ and can lead to an increase in challenging behaviours such as agitation, aggression and wandering. ${ }^{2}$ Such cognitive and behavioural changes can make it more time consuming for long-term care (LTC) facility staff to provide care for people with dementia ${ }^{3}$ and can lead to greater levels of stress. ${ }^{4}$ This can then have implications for the retention and recruitment of staff but also, more importantly, may negatively affect the quality of care provided to the person with dementia. ${ }^{5}$ Given these concerns, coupled with statistics that show dementia to be an increasingly prevelant condition, ${ }^{6}$ there is a need to understand how these challenging behaviours may be ameliorated. Such research may be particularly necessary in light of the increasing number of older people living in LTC in many countries. ${ }^{7,8}$ However, undertaking research with people with dementia and then more generally in LTC is often fraught with challenges. ${ }^{8,9}$ For instance, the level of cognitive decline and the presence of agitated behaviours may affect or compound consistent intervention implementation ${ }^{10}$ and make it difficult for the person with dementia to complete outcome measures. ${ }^{11}$ In addition, acute illness or death also makes maintaining a large enough sample size an ongoing struggle. ${ }^{12}$ When examining the challenges encountered in the LTC environment, inflexible facility routines, policies and practices, ${ }^{7,9}$ staff non-compliance with research protocols, ${ }^{7}$ high levels of staff turnover, and reduced staff-to-resident $\operatorname{ratios}^{9}$ have all been found to make research cumbersome. As a result, research conducted within LTC with both frail older people and, specifically, those with dementia has generally been understudied. ${ }^{8,13}$ This has particularly been the case for clinical or RCTs ${ }^{13,14}$ owing to the required rigour and extent of control over extraneous variables needed. 
The adoption of psychosocial treatments for dementia has received increasing research interest and the therapeutic use of music has been one approach that has gained popularity, ${ }^{15}$ showing efficacy in terms of reducing agitation, ${ }^{16-18}$ anxiety $^{17,18}$ and depression ${ }^{19}$ and in improving quality of life. ${ }^{20,21}$ However, many of these studies have lacked methodological rigour $^{22}$ and, thus, more stringently controlled trials are necessary to substantiate claims. The RCT, considered the 'gold standard' of clinical trials ${ }^{23}$, offers the rigour that many of the previous studies lack, with the central principles including: randomisation (random assignment of participants to intervention and control groups); manipulation (manipulation of the control and intervention); and control (measures taken to reduce the influence of extraneous variables including a comparable control group). ${ }^{24}$

In light of the issues outlined above, and the need for more clinical trials in LTC, this paper provides a timely critical discussion of the challenges encountered whilst conducting a RCT with cross-over-design. This discussion moves beyond previously published papers by reflecting on the challenges of using this design (a RCT) to answer research questions in this setting (LTC) and with this population (older people with dementia) within the one paper. The challenges critiqued and the ways in which we tried to overcome them are discussed according to the three principles of a RCT to keep experimental rigour at the forefront of the discussion and the research area.

\section{STUDY}

A RCT with a cross-over design was employed to explore the effect of a live group music program on agitation, emotion and quality of life of older people with dementia. ${ }^{25-28}$ The study followed the recommendations outlined in the Consolidated Standards of Reporting 
Trials (CONSORT) statement. ${ }^{29}$ An overview of each main aspect of the study is set out in Table 1.

[Table 1 here]

\section{CHALLENGES ENCOUNTERED}

\section{Randomisation}

\section{Sample and Setting}

The choice of LTC facility from which to conduct the research is an important first decision in the research process. The cooperation and flexibility of facility staff is paramount to the successful implementation of an intervention ${ }^{31}$ as often there is reluctance to take part owing to concerns that it may add to current workloads and interrupt ongoing activity schedules. ${ }^{8,12}$ In the RCT described here, two Research Assistants (RAs) employed by the University were based at each of the two facilities during implementation. Their role was to oversee the logistical aspects of the process, such as managing the transportation of participants to the intervention. This helped reduce the workload pressures on facility staff but also ensured that the success of the intervention was not reliant on facility cooperation or help. This proved especially important in one facility where, in the second half of the intervention, no facility assistance could be offered to researchers. Budgets are often exceptionally tight and there is typically only scope for employment of absolutely necessary personnel. However, it is recommended that the budget should allow for adequate RA support as this may safeguard against success of the research being dependent on facility assistance and their level of commitment.

The recruitment process presents a number of challenges to the research team including identifying and recruiting participants, obtaining consent and the formal enrolment 
of participants. As in our study, research undertaken within the LTC setting typically sees the care manager orchestrating the informed consent procedure. This process, however, is often lengthy and time-consuming, as staff do not always identify participants immediately and do not send out the consent packs until later still. In the music RCT, the consent process took around eight - ten weeks and this caused some delay to the start of the intervention. As such, it is recommended that a generous 12-week consent and recruitment period be allowed for. Furthermore, if multiple facilities are involved then a longer time-frame may be required as the speed and success of recruitment can vary, as was our experience.

Further delays to the consent and recruitment process can also be experienced because the onus, when the person has severe cognitive impairment, is primarily on proxy consent involving next of kin. ${ }^{31}$ This can pose challenges as family members can often think that their relative has health issues that are too severe to allow participation. ${ }^{12}$ In addition, the next of kin can sometimes only desire for their relative to take part in the intervention and not the control activity. This was an issue in our study where families often expressed that their relative would enjoy the music but not the reading activities. Researchers explained the importance of the control group in helping to determine the effectiveness of the intervention and also explained what the control activity would entail. This helped clarify concerns and in most cases consent was provided. However, this issue is often a challenge in studies involving a control group and can be an ongoing struggle.

The formal enrolment of participants needs to be carefully considered in terms of which residents are eligible and their characteristics. In determining the eligibility criteria, consideration should be given to whether participants from all areas of the LTC facility are to be included or only those from the Special Care Unit (SCU), high (nursing home) or low 
(assisted) care respectively. In the music RCT, participants were selected from all areas but the intervention/control activities were held in central facility locations. For one participant, movement from SCU to the intervention caused distress and agitation. It may be advantageous, therefore, for future studies to consider running interventions in separate areas of the facility so that movement does not differentially influence the measured effectiveness of the intervention. It is recognised, however, that this would have cost implications by resulting in the probable need for multiple sites to ensure adequate participant numbers.

Another important consideration is the extent to which participants are screened on outcome measures prior to formal enrolment. For instance, should there be a minimum score on outcome measures for study eligibility? In the music project, participants were eligible for inclusion based on their level of cognitive impairment, as assessed on the Mini Mental State Examination (MMSE), ${ }^{32}$ and a documented behavioural history of agitation/aggression on nursing records within the last month (See Table 1). Despite these pre-requisites, however, baseline scores on all outcome measures were low. The inclusion of such participants may mask or attenuate the efficacy of the intervention and, thus, in line with previous researchers ${ }^{16,23,33}$ we advocate future studies would benefit from more in-depth screening of participants prior to study commencement. Although this may compound the difficultly in finding eligible participants, it may help improve the reliability and validity of findings.

Finally, even though a RCT cross-over design helps reduce the number of participants to be recruited, ${ }^{34}$ it may also be advantageous to over-sample when undertaking research with older people, given that participant attrition is common. ${ }^{8}$ In the music study, participants were over-sampled by $10 \%$, and this was accurate for the study timeline and in accounting for the 
number of deaths ( $n=5,10.6 \%)$. Future researchers should consider over-sampling by at least $10 \%$ when studies are lengthy to maintain study power in the likely event of attrition.

\section{Manipulation}

\section{System characteristics of the LTC facility}

System characteristics of the LCT facility, such as the schedule of activities and daily routine, often present challenges in terms of when the intervention can be run. ${ }^{12}$ Academia often highlights the need to conduct research when the symptoms under investigation are most

prevalent to maximise the chances of detecting a significant effect. ${ }^{23,33}$ However, the LTC facility staff will typically dictate the times when the intervention can be held. For instance, in the music RCT the two LTC facilities were keen to hold the sessions in the morning, as activities were planned for the afternoon. This was regardless of when participants' agitation levels were at their peak. Researchers, when planning the timing of interventions, should always take account of the normal facility schedule and ensure it is workable within the facility. However, it is also worth pursing a discussion regarding the advantages of conducting the study when individual resident's symptoms under investigation are at their peak, as this may increase the chances of discovering a treatment effect and help better determine if the intervention will be of benefit to participants and care staff.

\section{Logistical issues}

When planning the implementation of the intervention and control groups, the location of the activities must be carefully considered. In our RCT, the music and reading control activities were held in an activities room in one facility and a chapel in the other. These rooms were selected by facilities, as no other areas were available, and required the transportation of participants to the rooms. In addition, the chapel was detached from the main facility 
buildings and involved a period of transportation outside. This was problematic when weather conditions were not amenable (i.e., raining) as this reduced willingness to attend sessions. It would be advisable, therefore, to select rooms that are located within the main facility building so as to control for such extraneous variables. However, it is recognised that space is an issue in many facilities and there are often only limited rooms from which to run the intervention.

The need for consistency during the implementation of the intervention and control is paramount and this includes promptness of start and end times. However, transporting participants to the activities in preparation for a punctual start can be difficult. For instance, in the music program there were some participants who became restless when brought to the room too early prior to the start of the session. For these participants, the RAs had to ensure that they were brought just before the sessions commenced, therefore resulting in some participants waiting for the activity longer than others. In addition, a number of participants required constant staff supervision, meaning that when brought to the venue, the RA had to stay with them. This resulted in fewer staff being able to help with the transportation of the remaining residents. The possible extraneous influence that different lengths of time waiting for the activity and the different ways that participants' mood and behaviour can be affected by transportation to the venue highlights the importance of careful logistical planning, as such influences need to be controlled in RCTs to ensure validity of findings. It may be advantageous for community volunteers at the facility to help with the transportation of residents. One facility in the music program had a bank of volunteers who were able to help and this was of great assistance. 


\section{Maintaining session attendance}

When undertaking any RCT, there will always be challenges in maintaining participant session attendance. However, when undertaking research with older people with dementia in LTC, this can be more of an issue because of the characteristics of the population being studied. In the music RCT, it was common for participants to refuse to attend a session, primarily because of ill-health and/or cognitive impairment (i.e., were agitated, confused or forgot). Some participants also just wanted to attend the music sessions and so refused to attend any reading activities. Facility staff also contributed to missed sessions by failing to get participants out of bed or by not asking the participant if they wanted to attend. This reinforces the importance of having the cooperation of facility staff, as it is beyond the realm of a research team to ensure that participants are up and dressed ready for the intervention.

\section{Control}

\section{Treatment fidelity}

Issues associated with treatment fidelity are of integral importance when delivering intervention and control activities as, ultimately, they can enhance the reliability and validity of the results. ${ }^{35}$ At the core of a good treatment fidelity strategy is the standardized procedures manual. ${ }^{36}$ A comprehensive manual was produced in our RCT and all staff involved in delivering the activities were expected to adhere to this manual. Four random spot checks in the intervention and control activities were undertaken by the research team to ensure that the sessions were being delivered consistently and in clarifying the role of the musicians and reading group facilitator (i.e., musicians were to encourage participation such as dancing but were not to lead behaviour such as getting up to dance first). Also important in maintaining treatment fidelity was the practical, role-based training in delivering the sessions and in 
working with older people with dementia. This suite of measures was effective in controlling the delivering of the sessions and similar steps are recommended for future research.

\section{Outcome measures and data collection}

Deciding what instruments and scales to use in a study can be a difficult task, especially given the wide array available. Four main outcome measures were employed in the live music program: the Rating Anxiety in Dementia Scale (RAID); ${ }^{37}$ the Dementia Quality of Life questionnaire (DQOL); ${ }^{38}$ the Geriatric Depression Scale (GDS); ${ }^{39}$ and the Cohen-Mansfield Agitation Inventory - Short Form (CMAI-SF). ${ }^{40}$ These measures were chosen because of their demonstrated reliability and validity but also because they varied in methodological approach and included proxy reports (care staff completed the CMAI) and self-report (RAs interviewed the person with dementia on the RAID, DQOL and GDS). By using a range of outcome measures a more holistic assessment can be sought. This is especially important in dementia-care research as often, as the severity of dementia develops, verbal communication can become more difficult. ${ }^{11}$ It is also useful as it offers insight into any discrepancies on outcome measures depending on the perspective of the person completing the measure. ${ }^{41}$

When choosing outcome measures it is also useful to consider using instruments that are comprised of subscales and not one global, overall score. ${ }^{11}$ Such scales account for subtleties in the effect of the intervention. This was the case for the DQOL 'belonging' subscale in the music RCT, where a significant effect was detected. If only a global score had been computed then a Type II error may have occurred. 


\section{Data analysis and handling missing data}

Determining the analysis framework is an important stage in research, as this can determine the results produced and the findings drawn. In the music study, an 'Intention-To-Treat' (ITT) analysis was undertaken; the 'gold standard'42 as all participants are included irrespective of the treatment actually received, thus avoiding overestimation of clinical effectiveness. ${ }^{43,44}$ When adopting such a framework a second decision needs to be made about handling missing data. A number of methods can be applied including imputing means, carrying the last score forward and single and multiple imputations. The last method is generally regarded as the most sophisticated approach seeing multiple plausible data sets computed and the results pooled. This was the method chosen for the music study and, as recommended, a sensitivity analysis was then undertaken to verify the results and confirm no differences according to approach. ${ }^{43,45}$ This saw parallel analyses conducted comparing results when missing data was addressed through multiple imputation, case mean substitutions or left as missing. As multiple imputation is becoming more common in statistical software, it is recommended that future researchers consider conducting ITT analysis using this method of imputation. However, if the use of multiple imputation is deemed appropriate, it should also be undertaken alongside some sort of sensitivity analysis to verify that the imputation is not distorting any significant effects.

\section{LESSONS FROM THE FIELD: CLINICAL CONSIDERATIONS}

Based upon our experiences of conducting the music RCT, future researchers may benefit from considering the following questions, outlined in Table 2, when designing and implementing similar RCTs.

[Table 2 here] 


\section{CONCLUSION}

The characteristics of older people with dementia and the LTC environment can make conducting research a challenge and, as such, this population subgroup is often understudied, particularly in terms of clinical or RCTs. In this paper we have critically discussed some of the difficulties that we faced whilst implementing a RCT involving a live music program with older people with dementia in LTC. It is hoped that this discussion provides a starting point for researchers designing similar RCTs and helps encourage a proactive approach in dealing with research-related challenges right from project conception.

\section{ACKNOWLEDGEMENTS}

This paper reports on a larger study funded by the National Health \& Medical Research Council, Australia (Grant ID 481929). The involvement of the sponsor was monetary and did not include support in the design, collection and analysis of data or the final report. The authors acknowledge support and contributions by RSL care staff, family and residents during the research process. 


\section{REFERENCES}

1 Australian Institute of Health and Welfare [AIHW]. Dementia in Australia: national data analysis and development. Canberra: 2007. Australian Institute of Health and Welfare, AIHW Cat No. AGE 53.

2 Boller FM, Verny M, Hugonot-Diener L, Saxton J. Clinical features and assessment of severe dementia: a review. European Journal of Neurology 2002; 9: 125-136.

3 Ory MG, Hoffman RR III, Yee JJ, Tennstedt S, Schulz R. Prevalence and impact of care giving: a detailed comparison between dementia and nondementia caregivers. The Gerontologist 1999; 39: 177-185.

4 Mott S, Kingsley B. Challenging behaviour: the issues of quality of care. In: Garratt RNS (eds), Nursing Older People: Issues and innovations. Sydney: MacLennan \& Petty Pty Ltd, 1999; 212-233.

5 Goergen T. Stress, conflict, elder abuse and neglect in German nursing homes: a pilot study amongst professional caregivers. Journal of Elder Abuse 2001; 13: $1-26$.

6 Ferri CP, Prince M, Brayne C et al. Global prevalence of dementia: a Delphi consensus study. Lancet 2005; 366: 2112-2117. 
7 Hall S, Longhurst S, Higginson IJ. Challenges to conducting research with older people living in nursing homes. BMC Geriatrics 2009; 9:(38).

8 Maas ML, Kelley LS, Park M, Specht JP. Issues in conducting research in nursing homes. Western Journal of Nursing Research 2002; 24: 373-389.

9 Mentes JC, Tripp-Reimer T. Barriers and facilitators in nursing home intervention research. Western Journal of Nursing Research 2002; 24: 918936.

10 Snyder M, Tseng Y, Brandt C et al. Challenges of implementing intervention research in persons with dementia: example of a glider swing intervention. American Journal of Alzheimer's Disease and Other Dementias 2001; 16: 5156.

11 Mittelman MS. Psychosocial intervention research: challenges, strategies and measurement issues. Aging \& Mental Health 2008; 12: 1-4.

12 Cohen-Mansfield J. Nonpharmacologic interventions for inappropriate behaviours in dementia: a review, summary and critique. American Journal of Geriatric Psychiatry 2001; 9: 361-381.

13 Working group on functional outcome measures for clinical trials. Functional outcomes for clinical trials in frail older persons: time to be moving. Journal of Gerontology: Medical Sciences 2008; 63A: 160-164. 
14 Jackson J, Mandel D, Blanchard J et al. Confronting challenges in intervention research with ethically diverse older adults: the USC Well Elderly II Trial. Clinical Trials 2009; 6: 90-101.

15 Bruer RA, Spitznagel E, Cloninger CR. The temporal limits of cognitive change from music therapy in elderly persons with dementia or dementia-like cognitive impairment: a randomised controlled trial. Journal of Music Therapy 2007; 44: 308-328.

16 Ledger AJ, Baker FA. An investigation of long-term effects of group music therapy on agitation levels of people with Alzheimer's Disease. Aging \& Mental Health 2007; 11: 330-338.

17 Raglio A, Bellelli G, Traficante D et al. Efficacy of music therapy in the treatment of behavioural and psychiatric symptoms of dementia. Alzheimer's Disease and Associated Disorders 2008; 22: 158-162.

18 Svansdottri HB, Snaedal J. Music therapy in moderate and severe dementia of Alzheimer's type: a case-control study. International Psychogeriatrics 2006; 18: $613-621$.

19 Ashida S. The effect of reminiscence music therapy sessions on changes in depressive symptoms in elderly persons with dementia. Journal of Music Therapy 2000; 37: 170-182. 
20 Clair AA, Bernstein B. A preliminary study of music therapy programming for severely regressed persons with Alzheimer's-type dementia. The Journal of Applied Gerontology 1990; 9: 299-311.

21 Rio R. Improvisation with the elderly: moving from creative activities to process-oriented therapy. The Arts in Psychotherapy 2002; 29: 191-201.

22 Boso M, Politi P, Barale F, Emanuele E. Neurophysiology and neurobiology of the musical experience. Functional Neurology 2006; 21: 187-191.

23 O’Connor DW, Ames D, Gardner B, King M. Psychosocial treatments of behaviour symptoms in dementia: a systematic review of reports meetings quality standards. International Psychogeriatrics 2009; 21: 225-240.

24 Taylor B, Kermode S, Roberts K. Research in nursing and health care: Evidence for practice ( $3^{\text {rd }}$ ed.). Victoria: Thomson, 2006.

25 Cooke M, Moyle W, Shum D, Harrison S, Murfield J. The effect of music on quality of life and depression in older people with dementia: a randomised control trial. Alzheimer's \& Dementia 2009; 5: e11.

26 Cooke M, Moyle W, Shum D, Harrison S, Murfield J. A randomized controlled trial exploring the effect of music on agitated behaviours and anxiety in older people with dementia. Aging and Mental Health in press. 
27 Cooke M, Moyle W, Shum D, Harrison S, Murfield J. A randomised controlled trial exploring the effect of music on quality of life and depression in older people with dementia. Journal of Health Psychology in press.

28 Harrison S, Cooke M, Moyle W, Shum D, Murfield J. Development of a music intervention protocol and its effect on participant engagement: experiences from a randomised controlled trial with older people with dementia. Arts \& Health in press.

29 Schulz KF, Altman DG, Moher D. CONSORT 2010 Statement: updated guidelines for reporting parallel group randomised trials. Annals of Internal Medicine 2010; 152:1-7.

30 American Psychiatric Association. Diagnostic and Statistical Manual of Mental Disorders (4 ${ }^{\text {th }}$ ed.). USA: American Psychiatric Association, 1994.

31 Finkel S, Cooler C. Clinical experiences and methodologic challenges in conducting clinical trials on the behavioural disturbances of dementia. International Psychogeriatrics 1996; 8:151-164.

32 Folstein M, Folstein S, McHugh P. Mini-mental state. Journal of Psychiatric Research 1975; 12: 189-198. 
33 O’Connor DW, Ames D, Gardner B, King M. Psychosocial treatments of psychological symptoms in dementia: a systematic review of reports meetings quality standards. International Psychogeriatrics 2009; 21:241-251.

34 Senn S. Cross-over trials in clinical research $\left(2^{\text {nd }}\right.$ ed.). Chichester: Wiley, 2002.

35 Resnick B, Ignuito P, Orwig D et al. Treatment fidelity in behaviour change research: a case example. Nursing Research 2005; 54:139-145.

36 Chambless D, Hollon SD. Defining empirically supported therapies. Journal of Consulting and Clinical Psychology 1998; 66: 7-18.

37 Shankar KK, Walker M, Frost D, Orrell MW. The development of a valid and reliable scale for rating anxiety in dementia (RAID). Aging and Mental Health 1999; 3: 39-49.

38 Brod B, Stewart A, Sands L, Walton P. Conceptualization and measurement of quality of life in dementia: dementia quality of life instrument (DQoL). The Gerontologist1999; 39: 25-35.

39 Yesavage JA, Brink TL, Rose TL et al. Development and validation of a geriatric depression screening scale: a preliminary report. Journal of Psychiatric Research 1983; 17: 37-49. 
40 Werner P, Cohen-Mansfield J, Koroknay V, Braun J. The impact of a restraint-reduction program on nursing home residents. Geriatric Nursing 1994; 15:142-146.

41 Cotrell V, Schulz R. The perspective of the patient with Alzheimer's Disease: a neglected dimension of dementia research. The Gerontologist 1993; 33: 205211.

42 Heritier S, Gebski V, Keech A. Inclusion of patients in clinical trial analysis: the intention-to-treat principle. The Medical Journal of Australia 2003; 179: 438-440.

43 Hollis S, Campbell F. What is meant by intention to treat analysis? survey of published randomised controlled trials. British Medical Journal 1999; 319: 670-674.

44 Kruse RL, Alper BS, Reust C, Stevermer JJ, Shannon S, Williams R. Intention-to-treat analysis: Who is in? Who is out? The Journal of Family Practice 2002; 51: 969-971.

45 Sterne J, White I, Carlin J et al. Multiple imputation for missing data in epidemiological and clinical research: potential and pitfalls. British Medical Journal 2009; 339: 157-160. 
Table 1 Overview of study

Methodological aspect Description of study

Design $\quad$ Randomised cross-over design with music intervention and reading control group, from October 2008 to March 2009.

Ethical approval granted by University human research ethics committee and a support statement provided by partner aged care organisation.

Sample $\quad 69$ assessed for eligibility and 47 formally enrolled into the research based on criteria: 1 . a confirmed diagnosis of early to mid stage dementia OR probable dementia (i.e., a cognitive impairment level of 12-24 on MMSE) OR features consistent with dementia of Alzheimer's type as per DSM-IV ${ }^{30}$ AND 2. a documented behavioural history of agitation/aggression on nursing/medical records within the last month. Consent provided by next of kin and, where possible, participants. The study’s biostatistician, blinded to participant identity, undertook randomisation process of treatment group allocation.

Setting Two mixed-gender LTC facilities North of Brisbane, Australia. Site A = 164 residents and Site B = 94 residents. Intervention and control activities ran for 40-minutes, 3 mornings a week for 8 weeks. After a 5-week 'washout' period, participants crossed-over into opposite activity and protocol repeated for another 8 weeks. Intervention was a live group music program involving 30-minutes of musician-led familiar song singing and 10-minutes of pre-recorded 
instrumental music. Reading control sessions included reading local news stories, short stories, telling jokes and quiz activities.

Data collection

Data analysis
Assessments at baseline, mid-point (wash-out period) and post-intervention on 4 outcome measures:

By RAs: RAID; DQOL; and GDS.

\section{By care staff: CMAI-SF.}

Data entered and analysed using the Statistical Package for the Social Sciences Version 17.0 (SPSS Inc., Chicago, IL, USA). Following a missing values analysis, an ITT framework was undertaken and missing values in outcome measures were addressed through a multiple imputation method. 
Table 2 Clinical considerations when planning a RCT with a psychosocial intervention involving older people with dementia in LTC

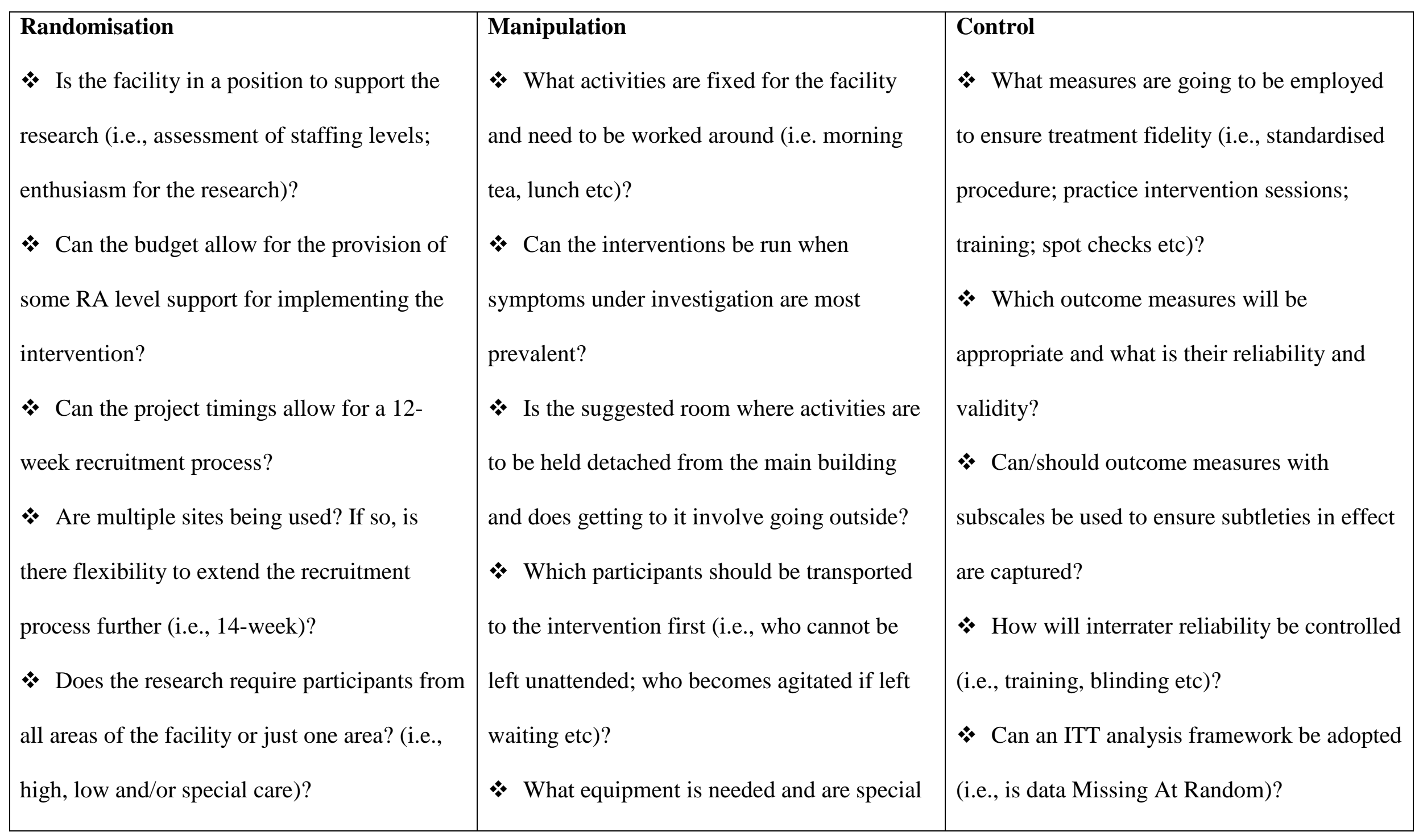




\begin{tabular}{|l|l|l|}
\hline an outcome measure necessary (i.e., is a & $\begin{array}{l}\text { requirements necessary (i.e., ease of } \\
\text { minimum level/assembly, level of noise etc)? }\end{array}$ & Can time for a sensitivity analysis of data \\
& $\begin{array}{l}\text { taking part in which activity and the need for } \\
\text { them to be ready if they wish to attend? }\end{array}$ & \\
\hline
\end{tabular}

